\title{
Infrared Reflectivity Spectra of Manganite Thin Films Grown on Different Substrates
}

\author{
A. Antonakos · E. Liarokapis • M. Filippi - W. Prellier • \\ G.H. Aydogdu • H.-U. Habermeier
}

Received: 10 November 2008 / Accepted: 10 November 2008 / Published online: 25 November 2008

(C) The Author(s) 2008. This article is published with open access at Springerlink.com

\begin{abstract}
We have measured the reflectivity infrared (IR) spectra of $\mathrm{R}_{1-x} \mathrm{Ca}_{x} \mathrm{MnO}_{3}(\mathrm{R}=\mathrm{La}, \mathrm{Pr})$ manganite thin films grown on different substrates $\left(\mathrm{SrTiO}_{3}(\mathrm{STO}), \mathrm{LaAlO}_{3}\right.$ (LAO) and $\mathrm{SrLaGaO}_{4}(\mathrm{SLGO})$ ) manganites over a wide frequency $\left(50-5000 \mathrm{~cm}^{-1}\right.$ ) range. In the Far IR (FIR) region the substrates dominate over the manganite spectrum. However, the previously observed infrared active modes or mode pairs could be identified. In the mid-IR (MIR) region, a characteristic insulating gap at $\sim 700 \mathrm{~cm}^{-1}$ is always present for all thin film studied, which shows substrate and thickness dependence.
\end{abstract}

Keywords Infrared · Manganites · Thin films

\section{Introduction}

Rare-earth manganites $\left(\mathrm{R}_{1-x} \mathrm{~A}_{x} \mathrm{MnO}_{3}\right.$, R: a trivalent rareearth ion; A: a divalent alkaline-earth ion) [1] attracted the scientific interest mainly due to their colossal magnetoresistance (CMR) effect [2]. Apart from their possible applications, manganites have been investigated extensively due to the complex physics involved [3, 4] and the rich phase diagram with many competing order parameters [5].

A. Antonakos $(\varangle) \cdot$ E. Liarokapis

Department of Physics, National Technical University of Athens,

15780 Athens, Greece

e-mail: tantonak@central.ntua.gr

M. Filippi · W. Prellier

Laboratoire CRISMAT, CNRS UMR 6508, ENSICAEN,

6 Bd Marechal Juin, 14050 Caen Cedex, France

G.H. Aydogdu $\cdot$ H.-U. Habermeier

Max-Planck-Institute for Solid State Research,

70569 Stuttgart, Germany
$\mathrm{La}_{1-x} \mathrm{Ca}_{x} \mathrm{MnO}_{3}$ is a characteristic example with the $\mathrm{LaMnO}_{3}$ as parent compound, which belongs to the family of rotationally distorted perovskites [6] and is an antiferromagnetic (AF) insulator. The bulk compound at the phase boundary with $x=0.5$ is a paramagnetic (PM) insulator and undergoes first a ferromagnetic (FM) transition and then a simultaneous AF and charge ordering (CO) transition at low temperature $\left(T_{c}=225-265 \mathrm{~K}\right.$ and $\left.T_{N}=130-160 \mathrm{~K}\right)$ [7].

$\operatorname{Pr}_{1-x} \mathrm{Ca}_{x} \mathrm{MnO}_{3}$ (PCMO) is another CMR compound with a rich phase diagram and a more distorted structure. At zero applied magnetic field it remains insulating for all $\mathrm{Ca}$ doping, while an antiferromagnetic (AF) to ferromagnetic (FM) metallic phase transition can be induced by the application of a magnetic field [8]. The charge ordering (CO) phase occurs at the doping range $0.3<x<0.7$. As the temperature range in which the CMR effect and the metal-toinsulator transition (MIT) are observed is close to room temperature (RT), these compounds are potential candidates for technical applications.

The phonon excitation spectrum has been studied in detail for the undoped $\mathrm{LaMnO}_{3}[9,10]$, which has below $\sim 750 \mathrm{~K}$ the Jahn-Teller (JT) distorted orthorhombic (Pnma) structure. A theoretical analysis of the complete set of phonon modes in the doped compounds is still missing.

In this paper, we present reflectivity IR results of $\mathrm{La}_{0.5} \mathrm{Ca}_{0.5} \mathrm{MnO}_{3}$ (LCMO) and PCMO $(0.4<x<0.6)$ thin films grown on different substrates that induce high in-plane compressive or tensile strains.

\section{Experimental Details}

We have studied $\mathrm{Pr}_{x} \mathrm{Ca}_{1-x} \mathrm{MnO}_{3}$ thin films grown in situ, using Pulsed Laser Deposition (PLD) on $\mathrm{SrTiO}_{3}$ (STO) and 
Fig. 1 The frequency-

dependent reflectivity of LCMO

at RT for different substrates in the $\mathbf{a}$ FIR and $\mathbf{b}$ MIR regions
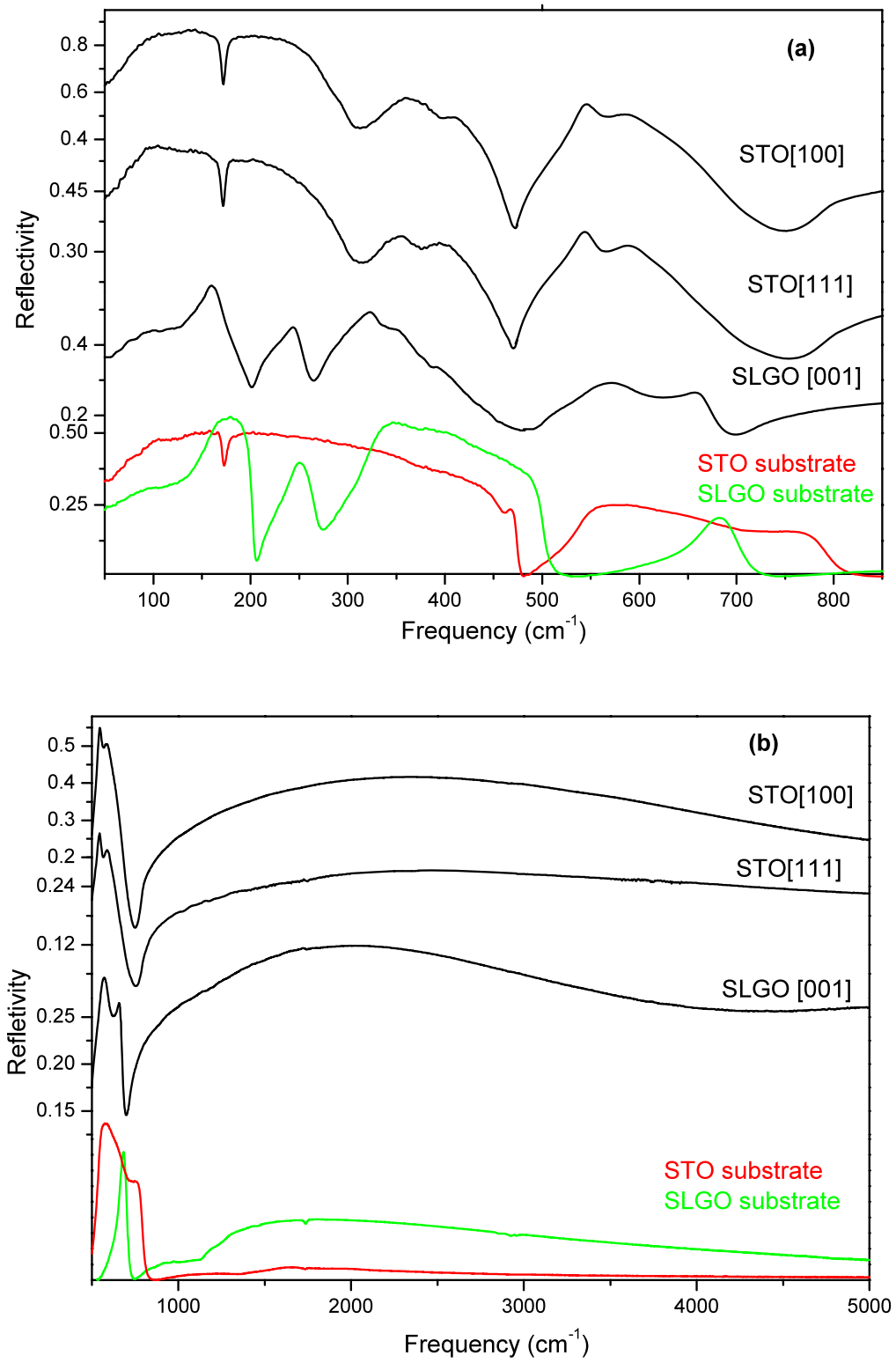

$\mathrm{LaAlO}_{3}$ (LAO) substrates (PCMO/STO and PCMO/LAO respectively). The LCMO films were deposited on (001) $\mathrm{SrLaGaO}_{4}$ (SLGO), (111) and (100) oriented planar $\mathrm{SrTiO}_{3}$ (STO) single crystal substrates by the PLD technique. A more detailed presentation of the growth procedure is described elsewhere [11, 12]. X-ray diffraction studies have shown that the films were single-crystal type and homogeneous as detected by transmission electron microscopy (TEM).

The Fourier transform IR (FTIR) spectra were recorded on a Bruker Optic IFS66v/S interferometer equipped with a reflectance unit. The spectra were collected with $2 \mathrm{~cm}^{-1}$ resolution, by averaging over a hundred of interferograms per spectrum.

\section{Results and Discussion}

Since LCMO and PCMO have the same Pnma structure with an orthorhombic unit cell containing four cubic units, 25 modes should be visible in the FIR spectra for both compounds. Approximately, the phonon spectra of manganites can be separated into external $\left(\sim 185 \mathrm{~cm}^{-1}\right)$, bending $\left(\sim 350 \mathrm{~cm}^{-1}\right)$, and stretching $\left(\sim 550 \mathrm{~cm}^{-1}\right)$ modes with respect to cubic (Pm3m) symmetry [13]. Depending on the ion size and doping concentration, these triply degenerate modes split into pairs of non-degenerate $(A)$ and doubly degenerate $(E)$ modes, and, moreover, they become broader and overlap [14]. Furthermore, due to the larger unit cell, additional modes emerge. 
Fig. 2 The frequency-

dependent reflectivity of PCMO

at RT for different substrates in

the $\mathbf{a}$ FIR and $\mathbf{b}$ MIR regions
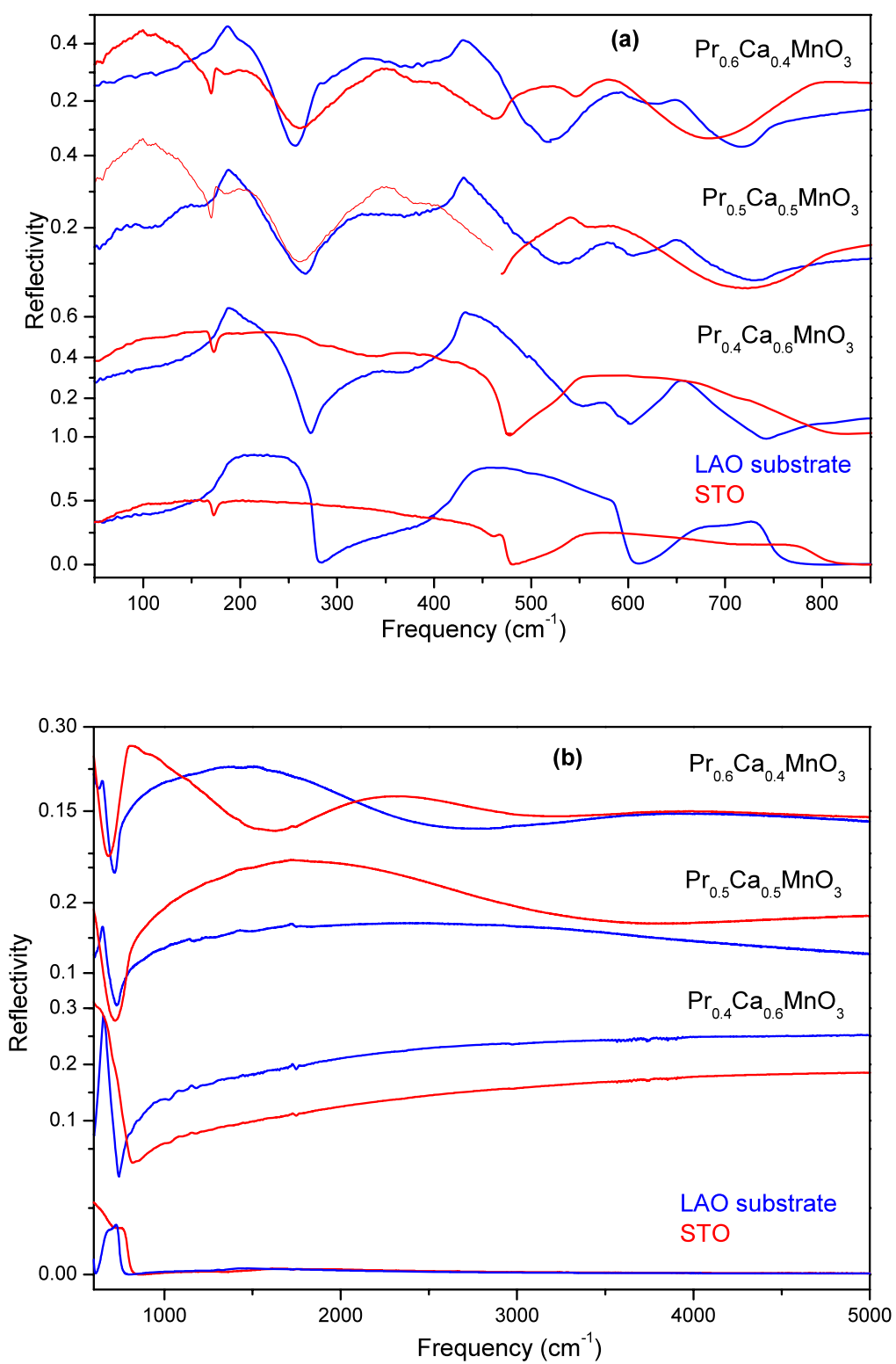

The reflectivity of LCMO is presented in Fig. 1 in the insulating PM (RT) phase. The substrates contribute considerably in the FIR region but little in MIR. As a result, we could not observe the $A_{u} / E_{u}$ doublet modes with TO frequencies at about $144 / 166 \mathrm{~cm}^{-1}$, which correspond to the vibrations of the La ions against the $\mathrm{MnO}_{6}$ octahedra along the $z$ - and $y(x)$-axes, respectively. The next doublet of $A_{2 u}$ and $E_{2 u}$ modes represents bond bending O-Mn$\mathrm{O}$ vibrations and is expected to appear at the frequencies $\sim 340 / 375 \mathrm{~cm}^{-1}$. This doublet is better observed for the thin film grown on STO(100) and STO(111), while they appear very weak and/or obscured by the SLGO substrate in the case of LCMO/SLGO thin films. Two strong bands appear at the $\sim 500-700 \mathrm{~cm}^{-1}$ region where the JT $F_{1 u}$ stretching mode is expected. The splitting of the $F_{1 u}$ stretching mode to two $A_{u}$ and $E_{u}$ modes has been also observed by others and has been attributed to the JT distortion, which induces different Mn-O lengths [13, 15]. The spectral resolution of phononic excitations in the films is superior to that in bulk LCMO samples and this is the reason we can resolve the stretching mode doublets in the Pnma symmetry. This has been already observed by Hartinger et al. [13] in the IR spectra of LCMO thin films comparing to polycrystalline spectra [16]; only three broad modes have been detected in the polycrystalline sample while the degeneracy has been removed in thin films.

In Fig. 1(b) a typical insulating behavior is observed with an energy gap of about $680 \mathrm{~cm}^{-1}$. This was expected as all thin films are PM insulators at RT, as found by magnetization measurements. The energy and the shape of the 
Fig. 3 The thickness-dependent reflectivity of LCMO/STO(100) at RT in the FIR and MIR regions

Fig. 4 The thickness-dependent reflectivity of LCMO/SLGO at $\mathrm{RT}$ in the FIR and MIR regions
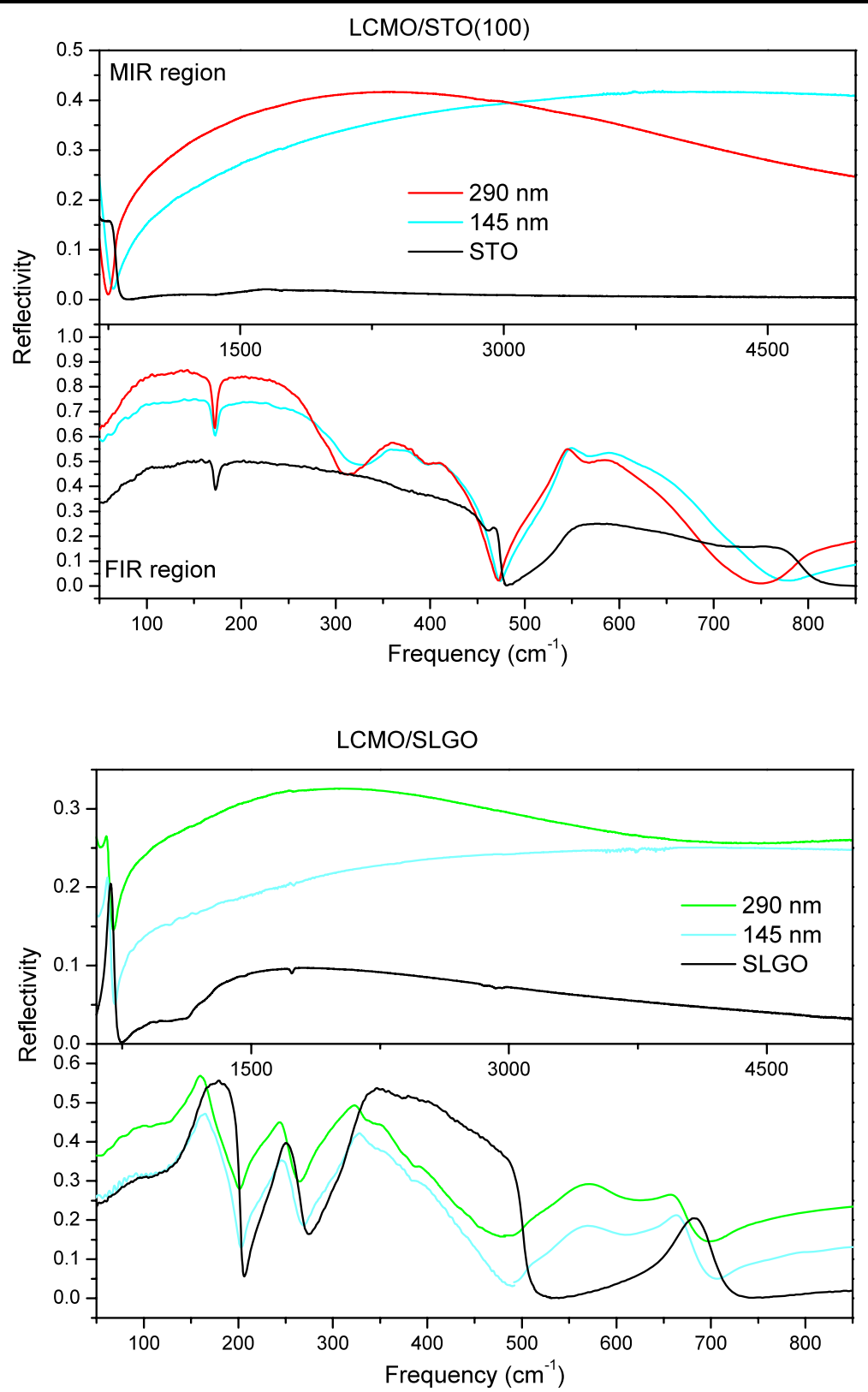

broad feature related with the insulating gap are affected by the different substrates. Finally, the reflectivity spectra of LCMO/STO(100) and LCMO/STO(111) are essentially identical, apart from a difference in the relative value.

The reflectivity of PCMO is presented in Fig. 2. These films are also PM insulators at RT, and since PCMO and LCMO have the same structure, we do not expect to see major differences. Indeed, the overall distinction between the reflectivity spectra of LCMO and PCMO is negligible (Figs. 1 and 2). One important difference is that, in the case of PCMO ( $x=0.5$ and $x=0.6$ ), the first doublet could be observed developed on the substrate spectrum in contrast with all other thin films. The most significant phonon difference concerns the stretching $F_{1 u}$ and the two $A_{u}$ and $E_{u}$ modes (Fig. 2(a)). These peaks appear at different frequencies, probably due to the different substrate strains and/or the orientations of the films.

The reflectivity of the MIR region is also affected by the different substrates and concentrations (Fig. 2(b)). Not only the frequency of the insulating gap changes but also a second broad band appears for $x=0.4$ centered at higher frequencies. Both peaks are strongly affected by the different substrates and temperature [17]. As already mentioned, several studies have shown that the PCMO thin films are phaseseparated with FM clusters competing with the AF/CO insulating ones. In addition, a recent resistivity study under the combination of pressure and magnetic field has shown that there are more than one insulating phases in the thin 
films [18]; an FM insulating phase, which mainly couples with the magnetic field, and an AF/CO one, which is suppressed by pressure. Therefore the two MIR broad bands could be attributed to those two phases.

In Figs. 3 and 4 we compare the effect of thickness on the reflectivity spectra. Since thickness is also regarded as an important factor for manipulating the properties of the films [12], differences should be expected in the MIR band if this is related with the presence of several phases (and gaps). Indeed, in all samples the intensity, the shape, and the characteristic energy of the insulating gap is affected by the thickness (Fig. 3, LCMO/STO(100) and Fig. 4, LCMO/SLGO) confirming the above assumption. The phonons in the FIR region are affected only in intensity; for the thicker films, the film contributes more in the film-substrate spectrum.

In conclusion, we have investigated the substrate dependence of the insulating MIR gap and the behavior of the IR phonons at room temperature. Our results indicate that the different substrates induce changes in the microscopic phases and in some cases in the structure of the thin films due to the different kind of strains. Although we did not obtain the optical conductivity from the Kramers-Kronig (KK) analysis due to the strong contribution by the substrate (substrate correction will be done in future work), thin films proved to be superior for the analysis of the phononic [13] and MIR spectra in the metallic manganites [19].

Acknowledgements Work funded by the E.C. STREP project No. 517039 "COMEPHS". The group of NTUA acknowledge additional financial support by Grant of NTUA "Peve2008".

Open Access This article is distributed under the terms of the Creative Commons Attribution Noncommercial License which permits any noncommercial use, distribution, and reproduction in any medium, provided the original author(s) and source are credited.

\section{References}

1. Ramirez, A.P.: J. Phys. Condens. Matter 9, 8171 (1997)

2. Jin, S., et al.: Science 264, 413 (1994)

3. Zener, C.: Phys. Rev. 82, 403 (1951)

4. Millis, A.J., et al.: Phys. Rev. Lett. 74, 5144 (1995)

5. Corey, J.M.D., Viret, M., von Molnar, S.: Adv. Phys. 48, 167 (1999)

6. Iliev, M.N., Abrashev, M.V., Lee, H.-G., Popov, V.N., Sun, Y.Y., Thomsen, C., Meng, R.L., Chu, C.W.: Phys. Rev. B 57, 2872 (1998)

7. Iliev, M.N., Abrashev, M.V.: J. Raman Spectrosc. 32, 805 (2001)

8. Tomioka, Y., et al.: Phys. Rev. B 53, R1689 (1996)

9. Fedorov, I., Lorenzana, J., Dore, P., DeMarzi, G., Maselli, P., Calvani, P., Cheong, S.W., Koval, S., Migoni, R.: Phys. Rev. B 60(11), 875 (1999)

10. Smirnova, I.S.: Physica B 262, 247 (1999)

11. Prellier, W., et al.: Appl. Phys. Lett. 77, 1023 (2000)

12. Aydogdu, G.H., Kuru, Y., Habermeier, H.U.: Mater. Sci. Eng. B 144, 123 (2007)

13. Hartinger, Ch., Mayr, F., Loidl, A., Kopp, T.: Phys. Rev. B 70, 134415 (2004)

14. Paolone, A., Roy, P., Pimenov, A., Loidl, A., Melnikov, O.K., Shapiro, A.Y.: Phys. Rev. B 61(11), 255 (2000)

15. De Marzi, G., et al.: Phys. Rev. B 68, 064302 (2003)

16. Kim, K.H., Gu, J.Y., Choi, H.S., Park, G.W., Noh, T.W.: Phys. Rev. Lett. 77, 1877 (1996)

17. Antonakos, A., Liarokapis, E., Filippi, M., Prellier, W.: J. Supercond. (2008, submitted)

18. Filippi, M., Auban-Senzier, P., Pasquier, C., Prellier, W.: Appl. Phys. Lett. 93, 142110 (2008)

19. Hartinger, Ch., Mayr, F., Loidl, A., Kopp, T.: Phys. Rev. B 73, 024408 (2006) 